\title{
Contraintes et libertés lexicales et grammaticales en langue anglaise des scientifiques
}

Jean-Louis Vidalenc

\section{(2) OpenEdition}

\section{Journals}

Édition électronique

URL : http://journals.openedition.org/asp/4276

DOI : 10.4000/asp.4276

ISSN : 2108-6354

\section{Éditeur}

Groupe d'étude et de recherche en anglais de spécialité

\section{Édition imprimée}

Date de publication : 1 octobre 1993

Pagination : 107-119

ISSN : 1246-8185

\section{Référence électronique}

Jean-Louis Vidalenc, «Contraintes et libertés lexicales et grammaticales en langue anglaise des

scientifiques », ASp [En ligne], 2 | 1993, mis en ligne le 27 février 2014, consulté le 20 avril 2019. URL http://journals.openedition.org/asp/4276; DOI : 10.4000/asp.4276

Ce document a été généré automatiquement le 20 avril 2019.

Tous droits réservés 


\title{
Contraintes et libertés lexicales et grammaticales en langue anglaise des scientifiques
}

\author{
Jean-Louis Vidalenc
}

1 1. Le thème retenu pour ce congrès, contraintes et libertés, s'insère tout naturellement dans l'œuvre poursuivie depuis plusieurs années ${ }^{1}$ en vue de l'affirmation d'une identité forte de l'enseignement en langues de spécialité. Celle-ci s'est, pour l'essentiel, forgée au fil des diverses rencontres du GERAS, dans une interaction entre les nouvelles technologies de l'enseignement et la didactique des langues².

2 À plusieurs reprises, quelques participants réguliers à nos colloques ont déploré le manque de passerelles entre les langues de spécialité et les Langues étrangères appliquées. Ces dernières auraient pu bénéficier, plus qu'elles ne l'ont fait, des apports à la rénovation pédagogique de l'enseignement aux non-spécialistes ${ }^{3}$. On n'a pas assez dit que c'est dans ce secteur des études anglaises que les innovations dans la manière d'enseigner ont été vraisemblablement le plus multiformes. Dans un autre effet pervers de non-communication entre anglicistes, la didactique de l'anglais scientifique a trop souvent ignoré les pistes de travail qu'auraient pu lui apporter les recherches lexicales et grammaticales, en amont des enseignements de traduction spécialisée en LEA4

3 Les orientations du DEA Langues de Spécialité ${ }^{5}$ constituent un encouragement à développer ces études sur le lexique et la syntaxe. Nous nous attacherons à développer une convergence qui est de nature à répondre aux besoins de formation de beaucoup d'étudiants.

4 Cet article rappellera dans un premier temps que des contraintes, plus fortes que dans bien d'autres domaines de l'anglicisme, composent l'environnement de l'enseignement et de la recherche sur la langue d'une macro ou d'une micro-spécialitét. Dans un second, nous montrerons que la démarche lexicogénétique permet à l'angliciste, dans l'approche de bon nombre de textes, de poser ses marques à côté de celles des scientifiques. Dans un 
troisième, nous nous intéresserons à quelques possibilités offertes par un travail sur la syntaxe, d'acquérir un espace de liberté encore plus significatif.

5 2.1. Qu'aucune recherche ne soit indépendante de son contexte institutionnel se vérifie tout particulièrement dans l'étude des langues de spécialité. Il serait tout à fait irréaliste que cet article se fixe pour objectif de procéder à un inventaire exhaustif des sémantismes de « langue de spécialité » dans la diversité des établissements et de rendre compte de l'hétérogénéité du vécu sociologique des enseignants. Ce serait toutefois faire l'impasse sur l'acquis de plus de dix années de débats au GERAS que de ne pas prendre en compte le caractère multiforme des contraintes qui pèsent sur la didactique de l'anglais scientifique dans bon nombre de structures administratives.

6 Les succès obtenus dans l'acquisition d'une représentativité par notre société savante, le fait que ses dirigeants aient acquis une autorité certaine dans les conseils scientifiques et des instances nationales de la Recherche ne doit pas faire oublier que les décisions institutionnelles dans la très grande majorité des enseignements de langue de spécialité sont prises par des responsables qui n'ont jamais effectué cette mission.

7 Dans les UFR de LCE, les contours des activités d'enseignement et de recherche en littérature, civilisation et linguistique sont, pour l'essentiel, délimités par des anglicistes. La situation est très variable en LEA, mais la rédaction du guide de l'étudiant résulte le plus souvent de décisions prises par des personnels ressortissant de la même section du $\mathrm{CNU}$ ou de sections voisines de celle des enseignants sur le terrain.

8 L'organisation, voire le contenu des cursus d'anglais pour scientifiques, même si les enseignants de langue, B ou C, sont parfois consultés, correspondent encore trop souvent à des équilibres entre enseignants A non anglicistes. Dans une communication sur la recherche envisageable en amont de la didactique de la langue, on ne peut faire abstraction de cette contrainte extrêmement lourde qui pèse sur un très grand nombre de collègues.

9 2.2. La contrainte qui apparaitt rapidement à tous ceux qui souhaitent réfléchir sur la langue de spécialité au-delà de la pratique d'un département, d'une UFR, voire des divers regroupements d'enseignants sur la base d'affinités, réside dans l'extrême diversité des contenus réels des enseignements de langue hors des filières $\mathrm{LCE}^{7}$. L'hétérogénéité du sémantisme de "langue de spécialité » ne facilite guère la compréhension et les coordinations entre enseignants. Ce n'est qu'au fil ces colloques qu'a pu se développer l'intercompréhension des vécus de chacun, qui a permis à la réflexion du GERAS de progresser.

10 2.3. Une troisième contrainte, très sous-estimée, consiste dans le très large spectre des besoins langagiers et, par conséquent des enseignements à mettre en place, dans les formations destinées aux non-spécialistes. Envisageons le cas d'un cours pour chimistes de Clermont-Ferrand ${ }^{8}$ conçu en fonction d'un séjour de travail de quelques semaines à Birmingham où existe une tradition comparable de liens entre laboratoires universitaires et industrie du caoutchouc. La difficulté essentielle ne réside pas dans l'inventaire des desiderata lexicaux et syntaxiques des comités de rédaction des revues où publient les uns et les autres. On peut la trouver davantage dans l'éventail des sujets de conversation possibles autour de la paillasse et plus encore dans les tea breaks et dinner parties du Staff Club. Là, il n'est pas impossible qu'on lui demande son avis sur une sortie possible au restaurant chinois, les charmes d'un week-end dans les Cotswolds où se déroule le dernier roman de David Lodge. 
11 On ne doit pas oublier la contrainte pédagogique que représente le décalage entre la multiplicité des tâches langagières et l'horaire habituel des enseignements aux nonspécialistes. Pour nous limiter à l'exemple de la compréhension des interventions dans un congrès scientifique, les difficultés sont largement au niveau de celles traitées dans les manuels qui font autorité sur la question ${ }^{9}$. Cette diversité des besoins en anglais des chercheurs scientifiques ne doit surtout pas servir de point d'appui rhétorique pour justifier l'absence dans des cours d'anglais de tout document extrait d'une bibliothèque de recherche. Dans notre communication au congrès du GERAS de Montpellier ${ }^{10}$ nous avons montré que dans l'éventail des tâches possibles, c'est sur le conseil à la rédaction d'articles que nous attendent d'abord, nos collègues scientifiques. difficilement envisager de trouver des effets positifs à certaines, la très grande variété des demandes de formation en langues nous semble avoir contribué à construire l'identité de l'enseignement de l'anglais à des scientifiques. Si ses missions vont au-delà de l'apprentissage de la traduction et de la rédaction, ces deux savoirs n'en occupent pas moins une place significative.

13 3.1. Cerner le domaine lexical de la micro-spécialité cible de l'enseignement constitue une étape indispensable avant de rédiger ou de traduire. Cela nous paraît aussi une manière pour l'enseignant d'affirmer sa liberté. En faisant appel à des méthodes de travail utilisées par des anglicistes, en montrant qu'elles apportent leur contribution à la progression des connaissances, il s'avère possible de construire une image positive de l'enseignement de la langue de spécialité.

14 Nous ne traiterons pas ici de la détermination de l'aire lexicale couverte par une microspécialité, on considérera que partir des cinq ou six revues de langue anglaise les plus citées dans la bibliographie du DEA ciblé peut constituer un corpus raisonnablement représentatif.

Les participants réguliers aux travaux du GERAS ${ }^{11}$ peuvent prendre appui sur une tradition orale de travail en commun, qui recouvre un nombre important de ces microspécialités. On peut ainsi estimer que le nombre moyen de lexies spécifiques se situe entre cent cinquante et trois cents.

16 Il faut gérer la contrainte qui fait que le sémantisme précis de la plupart des lexies spécialisées n'est totalement accessible qu'à ceux qui maîtrisent la discipline de l'intérieur. Une démarche interdisciplinaire permet, si elle est bien menée de mettre en valeur les apports des uns et des autres. Elle permet donc à l'angliciste de prendre en charge sa part de liberté.

17 Citons un exemple canonique de l'histoire des sciences. S.J. Gould explique que pour comprendre coadaptation, il faut avoir lu trois articles dont il donne les références (Dobzhansky 1948 ; Mayr 1963 ; Wallace 1968). L'enseignant d'anglais intéressé par son sujet peut constituer un inventaire des lexies et des problèmes qu'elles soulèvent, le biologiste prend le relais de l'explication au moment où le sémantisme résulte du consensus d'une communauté scientifique.

18 Contrairement à ce que croient beaucoup de ceux qui regardent les langues de spécialité de l'extérieur, les lexies d'apparence «barbare » qui apparaissent dans les articles très spécialisés, endoglycosydase ou phosphotyrosyl par exemple, posent peu de problèmes aux traducteurs. Dès qu'elles sont recensées sur les bases de données lexicales ou dans les dictionnaires spécifiques, elles sont remarquablement non ambiguës. On pourrait citer 
des pages d'exemples, mais il nous apparaîtrait peu valorisant de borner la contribution d'un angliciste au classement par ordre alphabétique des lexies ne figurant pas dans le Shorter. Les traducteurs débutants, à Bac +4 et $\mathrm{Bac}+5$, apprennent rapidement que les difficultés essentielles portent sur les mots qui ont l'air d'être des mots de tous les jours et dont le sémantisme scientifique ne peut être détecté par une procédure informatisée. Nos étudiants ont eu plus de mal à cerner netted dans un texte sur l'évolution que messenger ARN.

19 Si l'intersection lexie/concept doit, être gérée par les chercheurs scientifiques, nous allons constater que des zones d'ombre dans ce qui pourrait apparaître de la responsabilité de l'angliciste subsistent dans l'étude de quelques matrices lexicales ${ }^{12}$. L'affixation, la conversion, les métaphores et métonymies offrent un espace de travail et donc de liberté à qui veut s'intéresser à ces problèmes.

20 3.2.1. Si l'apport du vocabulaire des sciences à l'anglais dans son ensemble n'est pas le sujet essentiel du travail de Jean Tournier, il n'en apporte pas moins de nombreux éléments qui permettent d'en évaluer l'importance quantitative, y compris dans les dictionnaires généraux sur lesquels son étude est fondée. Dans le chapitre sur l'affixation, par exemple, près de quatre cinquièmes des préfixes et suffixes (recensés pourtant dans des dictionnaires non spécialisés) apparaissent exclusivement dans les textes de sciences expérimentales et de médecine.

21 C'est par exemple le cas des préfixes anatomo, entero, haemo, immuno, pneumo, rhino, somato, uro, etc., et des suffixes aneous, ectomy, genic, phagy, ulous. La création continue de nouvelles molécules s'accompagne d'un mécano lexical utilisant l'affixation. Certaines des règles du jeu appartiennent à la science (la suffixation en ase des enzymes en est un exemple) mais le lexicologue de formation littéraire peut avoir accès à une partie non négligeable du dispositif.

3.2.2. À ce stade, l'enseignant de langues peut amorcer que ce soit chez les futurs traducteurs ou chez les futurs chercheurs scientifiques une réflexion sur la néologie. Cette discipline nouvelle qui a été maintes fois exposée dans de nombreux colloques d'anglicistes par John Humbley ${ }^{13}$ nous parait une des multiples voies envisageables pour une recherche originale dans une micro-spécialité.

L'histoire des sciences fournit des repères exacts permettant de situer l'origine d'une lexie et de déterminer son parcours. Lorsque le prix Nobel, Murray Gell-Man a baptisé en 1963 Quark la particule qu'il venait de découvrir, on a pu observer une quasisynchronisation entre une découverte expérimentale et l'apparition d'une nouvelle lexie. Ce processus est à la base d'un très grand nombre de créations lexicales qui entrent après quelques années dans les dictionnaires généraux. La réminiscence de Finnegan's wake illustre de manière particulièrement flagrante - ce qui lui vaut d'être souvent citée dans l'histoire des sciences - la diversité des sources d'inspiration des chercheurs dans la créativité lexicale. On peut regretter la disparition du premier nom des pulsars, little green men mais d'autres créations ludiques ont droit de cité dans le vocabulaire des sciences. Dans le film consacré au physicien Hawking par Channel 4, Wheeler raconte qu'il a inventé black hole au cours d'une conférence (1967) parce qu'il trouvait fastidieux de répéter pour la énième fois, la description plus scientifique du phénomène.

3.2.3. Ces trois anecdotes nous permettent d'aborder un point qui pose problème aussi bien aux traducteurs qu'aux chercheurs français qui se préparent à écrire un article en anglais. On le trouve dans la divergence des habitudes de rédaction entre le français et 
l'anglais. Les systèmes de traduction-machine utilisés par les services de traduction des Communautés Européennes à Bruxelles ${ }^{14}$ permettent de mesurer que la version française d'un article compte plus de signes que la version anglaise. Au-delà de différences dans la longueur des mots, il faut prendre en compte une différence de mode de fonctionnement. Le chercheur anglo-saxon utilise volontiers une métaphore hardie (par exemple les molécules chaperons en biochimie) ou un terme qui paraîtrait outrancièrement familier à son homologue français. Ainsi, Barbara Mc Clintock a eu un prix Nobel pour sa découverte des shrunken genes. On ne peut dire que cette lexie se situe dans le même niveau de langue que l'équivalent français : "gène codant pour la sucrose synthétase ». On peut observer un second étage de ce processus d'appropriation par les chercheurs, lorsque la seconde étape de la création lexicale appartient au registre familier. Ainsi les molécules découvertes par le chimiste Buckmaster Fuller, ont été nommées dans un premier temps Buckmasterfullerines, avant de devenir tout simplement bucky balls. Cette tendance de l'anglais est bien connue depuis les premières traductions de Jerome K. Jerome avec son housemaid's knee qui devient hygroma du genou. Plus récemment en écologie, le gaz à effet radiatif important est en anglais key greenhouse gas.

3.2.4. La siglaison apparaît comme une matrice lexicale particulièrement productive en langue scientifique. Même si là aussi, les bases de données lexicales s'imposent face aux dictionnaires spécialisés classiques grâce à la rapidité avec laquelle elles intègrent les néologismes, la gestion des acronymes peut se révéler délicate si l'enseignant doit gérer plusieurs micro-spécialités ${ }^{15}$. Les sigles relativement connus comme DNA ne posent pas de véritable difficulté au traducteur. Certains comme radar sont tellement intégrés à la langue quotidienne que beaucoup de locuteurs ne peuvent en reconstituer l'origine. Le cas des sigles plus récents comme wimps (weakly interactive massive particles) pose problème en particulier en traduction assistée par ordinateur. L'ingéniosité des chercheurs qui mettent un point d'honneur à trouver un sigle prononçable ne crée pas d'interférences mais ils ne mesurent pas toujours toutes les conséquences de la diffusion massive dans les revues d'un bloc de caractères déjà répertorié dans les dictionnaires les plus en usage.

3.3.1. La gestion de ces phénomènes de créativité lexicale a considérablement progressé en particulier grâce à l'analyse informatisée de corpus de grande taille. Lors de congrès précédents du GERAS, John Sinclair, Antoinette Renouf et Patrick Hanks ont mis en évidence quelques-unes de ces avancées ${ }^{16}$.

Une des interrogations des rédacteurs d'un dictionnaire et de leurs lecteurs avertis porte sur la nature des modifications du sémantisme d'un terme du vocabulaire scientifique lorsqu'il est intégré aux préoccupations quotidiennes d'une partie importante de la communauté de langue. Une proportion significative de ceux qui emploient big bang, oncogene, retrovirus sait que ces lexies ont été créées dans un contexte scientifique précis mais n'a pas la formation lui permettant de le maîtriser. Pour des raisons tout à fait compréhensibles - les problèmes de santé de tout un chacun fournissant un sujet de conversation - cette vulgarisation guette particulièrement le vocabulaire médical. Cette perte de scientificité rapide nous parait une des différences importantes entre le lexique de la biochimie et celui de la médecine ${ }^{17}$. Si on avait la possibilité de faire un comptage précis des emplois de calcium, on s'apercevrait vraisemblablement que chimistes et biochimistes représentent un pourcentage très faible des utilisateurs effectifs de la lexie, loin derrière des collectivités de patients inquiets. Ces phénomènes peuvent être gérés par des procédures précises d'analyse de corpus. Un traitement terminologique 
informatisé paraît envisageable. Ils ne sont pas sans pertinence pour la formation de traducteurs.

3.3.2. Métaphores et métonymies représentent les matrices lexicales vraisemblablement les plus insaisissables et les moins prévisibles. Des chercheurs français qui lisent les revues anglo-saxonnes tous les jours nous ont fait part de leur perplexité et de leur amusement devant l'exubérance de la créativité métaphorique de leurs homologues anglophones. Peu de physiciens français auraient spontanément eu sous la plume dans une revue ayant la crédibilité de Nature:

This would have been the kiss of death for the isolation strategy used by Nakanishi's group.

Dans les échanges de congrès qui sont partie intégrante du fond culturel de l'histoire de la physique on peut citer :

T'Hooft's work changed the Weinberg Salam frog into an enchanted prince.

Rappelons que Weinberg et Salam, pour ces travaux assimilés à un spécimen de batracien, avaient obtenu le prix Nobel.

On peut certes considérer que ces exemples, s'ils mettent bien en évidence une tendance lourde de la langue anglaise des scientifiques, ont une valeur pédagogique en tant que cas limites. Néanmoins, une analyse de corpus ou un travail en amont de la traduction machine marquera toujours un temps d'arrêt avant de pouvoir intégrer des créations métaphoriques au dispositif d'ensemble.

Ainsi, il n'était pas prévisible qu'un chercheur ayant à décrire ce qu'il avait observé au microscope électronique parle de leucine zippers, qu'un autre parle de son travail :

It began modestly as a field study to flesh out some chapters in a doctoral thesis. ${ }^{18}$ ou qu'un troisième écrive dans un livre :

We can walk a large distance across animal space.

33 On peut estimer que le caractère éminemment non prévisible de la création lexicale métaphorique qui permet d'envisager dans l'extrême diversité des micro spécialités représentées dans les établissements d'enseignement supérieur, d'innombrables espaces de liberté pour un nombre important d'enseignants de langue de spécialité.

Ce couple métaphore/métonymie est important pour notre propos en tant que matrice lexicale productive, dans une langue qui a été utilisée comme corpus en amont d'expérimentations sur la traduction machine. Il met en évidence le processus spontanément créatif de la langue naturelle et une des zones de difficultés essentielles dans la traduction scientifique ou l'apprentissage de la rédaction d'articles dans les revues spécialisées.

4.1. La recherche sur les phénomènes syntaxiques dans la diversité des spécialités scientifiques possibles doit pouvoir mettre encore plus en valeur les techniques de travail propres à l'anglicisme. Si les phénomènes lexicaux se prêtent particulièrement à une concertation avec les spécialistes scientifiques du domaine, un échange de vues autour de la recherche en syntaxe ne parait devoir intéresser que les informaticiens travaillant sur l'interface avec la langue naturelle. La recherche sur le fonctionnement de points de grammaire dans la diversité des micro-spécialités apparaît donc pour l'enseignant de langue de spécialité comme un lieu privilégié de responsabilité et de liberté.

On peut penser que la syntaxe a moins souffert que le lexique d'effets de tribune sur la simplicité apparente de la langue anglaise des scientifiques. La consultation de deux ouvrages récents conçus en fonction des épreuves de l'oral du CAPES, Faits de Langue Faits 
de Discours de Claude Delmas et al. et Réussir le Commentaire Grammatical de Textes de Jean Rémi Lapaire et Wilfrid Rotgé montre que la syntaxe peut transformer des phrases d'apparence simples en une suite de pièges. Par exemple, il n'existe pas de traduction unique de :

we are certainly making some progress.

Les jeux de l'énonciateur sur BE + ING et SOME, l'intonation rendent complexe compréhension et traduction d'un énoncé qui serait susceptible d'apparaître au cours d'une conversation dans un laboratoire.

4.2.1. Pour que cette liberté de l'enseignant de langues de spécialité puisse s'exercer dans le domaine syntaxique, il faut que les échanges avec les chercheurs en grammaire ne fonctionnent pas à sens unique. Les corpus de langue anglaise des scientifiques ont beaucoup apporté aux études sur la langue, en particulier comme il a été mentionné plus haut à l'époque des premiers travaux sur la traduction automatique. Les travaux de Guy Bourquin et du CELTA de Nancy ${ }^{19}$, en particulier, montrent bien que l'examen minutieux de milliers de pages de vulgarisation scientifique a apporté autant, voire plus, à la réflexion linguistique qu'à la composante informatique.

Nous nous bornerons faute de place à amorcer un débat qui fera l'objet d'une étude plus longue. Il nous semble intéressant de citer quelques exemples où le mode de fonctionnement de la spécialité scientifique et le sémantisme des lexies spécialisées met particulièrement en évidence le fonctionnement du marqueur grammatical.

4.2.2. La question de l'antériorité possible du prétérit par rapport au plus-que-parfait apparait comme une pomme de discorde dans des communautés de grammairiens. Dans l'exemple suivant :

Some paleontologists had argued that the sauropods arose directly from triassic hecondontians.

Il apparait peu raisonnable que les paléontologues aient pu discuter de l'origine de certains dinosaures avant qu'ils n'aient apparu sur terre.

4.2.3. La traduction des modaux apparaît souvent comme un autre secteur à risques. Ils rendent compte de la manière dont l'énonciateur envisage les chances de réalisation du prédicat. MAY est considéré comme exprimant l'équipossibilité, toutefois l'association MAY ...BUT apparaît comme un cas limite dans la mesure ou on peut situer la probabilité à $100 \%$. L'exemple habituellement cité est dû à Henri Adamczewski :

She may be pretty but she is a poor typist.

43 Dans notre corpus, on trouve sous la plume de Richard Dawkins, un des biologistes qui a forgé la théorie du gène égoïste, une des théories post darwiniennes les plus débattues dans la communauté scientifique :

Our genes may instruct us to be selfish but we are not obliged to obey them all our lives.

Dans son livre, le taux de probabilité de cohésion entre gene et be selfish est de $100 \%$, il se vérifie donc que le contenu sémantique balise la zone de déploiement du marqueur.

4.2.4. Si la composante anaphorique de l'article défini THE se trouve chez tous les bons auteurs, les exemples d'anaphores indirectes ou implicites apparaissent souvent les plus difficiles à gérer par les étudiants. L'exemple standard est probablement celui de Hawkins :

Bill worked at a lathe, the machine stopped. 
Ce n'est pas la grammaire des grammairiens qui s'avère déterminante dans l'emploi de l'anaphore, elle ne peut que renvoyer à la connaissance qu'a l'énonciateur de l'univers externe, de l'encyclopédie. En effet, le sémantisme de lathe étant un sous-ensemble de celui de machine, on se trouve devant un cas d'école d'anaphore indirecte.

Nous retrouvons ce rôle de renvoi dans un énoncé où les connaissances à maîtriser sont de la biologie animale accessible à tout lecteur intéressé :

Blackheaded gulls nest in large colonies, the nests being only a few feet apart. When the chicks first hatch out, they are small and defenceless and easy to swallow.

On peut observer ici un jeu double de l'anaphore indirecte entre nest verbe collectif et nest nom individuel, une autre entre nest verbe cause et chick nom conséquence.

4.2.5. Les quelques points que nous avons abordés ici montrent que trop souvent les marqueurs syntaxiques peuvent bloquer la compréhension de l'anglais des étudiants en sciences et se révéler source d'erreurs chez les apprentis traducteurs. Dans une perspective plus théorique, la confrontation entre le jeu du marqueur et des connaissances externes à l'énonciateur paraît devoir non seulement fournir un champ de liberté significatif à l'enseignant mais surtout permettre de contribuer à approfondir un des grands domaines des études anglaises.

0 En conclusion, la palette très large de besoins pédagogiques en enseignement de l'anglais à des scientifiques et la multiplicité des réponses sur le terrain ne va pas dans le sens d'une réponse unique à la question fondamentale de création de voies de passages des contraintes en direction de la responsabilité et la liberté de l'enseignant.

51 Que les mêmes textes puissent servir de point d'appui à l'apprentissage de la rédaction d'un article scientifique et à celui de la traduction spécialisée va dans le sens de la construction de passerelles entre diverses problématiques de l'enseignement et de la recherche. L'enseignement en langues de spécialité qui a beaucoup apporté dans l'application didactique des nouvelles technologies de l'enseignement doit pouvoir bénéficier en retour des contributions récentes aux études sur le lexique et la syntaxe.

C'est en assumant le défi que constitue l'analyse des phénomènes de nombreuses microspécialités et en prenant appui sur cette recherche pour répondre à la diversité des besoins de formation en langue contemporaine que beaucoup d'anglicistes vont pouvoir affirmer leur liberté et une présence dans le domaine des études anglaises.

\section{BIBLIOGRAPHIE}

Adamczewski, H. et J.-P. Gabilan. 1992. Clefs pour la grammaire anglaise. Paris : Armand Colin. Bourquin, G. et al. 1993. «États des recherches en traduction automatique au CELTA ». In Manuel d'Introduction à la Traduction Automatique. Paris : AUPELF.

Delecroix M. et J. Humbley. 1987. Pratique raisonnée de la compréhension accélérée de l'anglais oral. Paris : Hachette.

Delmas et al. Faits de langue, faits de discours. 1992. Courbevoie : Éditions de l'Espace Européen. 
Greven, H. et M.H. Greven. 1985. La compréhension orale en anglais Rouen : CRDP.

Lapaire, J.R. et W. Rotge. 1991. Linguistique et grammaire de l'anglais. Toulouse : Presses Universitaires du Mirail.

Laurian, A.M. (dir.). 1987. Contrastes Numéro spécila « La traduction automatique aspects européens » octobre.

Sinclair, John (dir.). 1987. Looking up. Glasgow : Collins.

Tournier, Jean. 1985. Introduction descriptive à la lexicogénétique de l'anglais contemporain. Genève : Slatkine.

Vidalenc, J.-L. 1990. «Organisation du sens, débat de validité de la prédication et discours scientifique ». In L'organisation du sens domaine anglais, recueil en l'honneur de Jean Lavédrine. St Étienne : CIEREC, 151-163.

\section{NOTES}

1. L'action menée depuis près de vingt ans par les animateurs du GERAS, le Président Michel Perrin, Jean-Marie Baïssus, Hubert Greven, Anne Pradeilles, Michelle Rivas, etc. a permis de constituer un champ disciplinaire d'enseignement et de recherche à côté des trois voies plus anciennes de l'anglicisme que sont littérature, civilisation et linguistique.

2. Nous renvoyons le lecteur aux actes des différents colloques du GERAS et tout particulièrement au numéro 1 de la revue ASp (mars 1993).

3. Nous ne développerons pas ici les différences de vécu des enseignants et les retombées de phénomènes institutionnels qui selon nous (rappelons que nous avons travaillé dix ans en service commun de langues en université scientifique et huit en LEA) expliquent le faible taux d'apport réciproque de ces deux secteurs d'enseignement.

4. Nous pensons tout particulièrement aux dispositifs associant enseignement et recherche en maîtrise, DEA et DESS, de Philippe Thoiron à Lyon 2 (anglais biomédical) et Daniel Gouadec à Rennes 2 (anglais informatique). À une plus petite échelle, nous avons mis en place à Pau un enseignement de maîtrise de traduction scientifique, articulé sur l'analyse linguistique de textes d'histoire des sciences.

5. Ce DEA national multi sceaux (Bordeaux, Montpellier) de langue des spécialités, créé sous l'impulsion de Michel Perrin, est animé par l'équipe de direction du GERAS, cf. note 1.

6. Nous appelons macrospécialité une grande discipline, physique, médecine, biologie végétale, etc. ; nous employons microspécialité pour définir (ce qui nous paraît d'ailleurs plus accessible à l'analyse linguistique) une discipline correspondant à la pratique d'un laboratoire, d'un DEA ou à un nombre limité de revues.

7. Le caractère national du CAPES impose, au-delà des diversités locales, une certaine homogénéité à ces enseignements.

8. Nous remercions un chimiste clermontois, dont nous avons malencontreusement oublié le nom, pour son témoignage recueilli lorsque nous avons rendu visite à John Sinclair et à l'équipe Cobuild. Rappelons que David Lodge a enseigné la littérature anglaise à l'université de Birmingham (qui devient Rummidge dans ses romans).

9. On lira avec profit Greven H \& M.H. (1985) et Delecroix \& Humbley (1987).

10. Un apport de l'analyse du discours à l'apprentissage de la rédaction d'articles scientifiques, l'étude des phénomènes de connexion M. Perrin, Ludmilla Delorme et J.-M. Baïssus (dir.) ASp 1 1993. 
11. Ce point a été mis en souligné par J.-M. Baïssus, lors de la présentation de cette communication. L'expérience de la gestion des différentes composantes (enseignement, recherche, administration) de l'interdisciplinarité dans les différentes strates d'anglais scientifique mériterait des développements allant bien au-delà des quelques pages d'un article.

12. La thèse que Jean Tournier (1985) a mis en évidence les mécanismes de base de production des lexies. Cette mise en évidence se révèle particulièrement précieuse dans la formation des traducteurs en particulier dans la gestion des néologismes.

13. Directeur du Centre de terminologie et de néologie du CNRS.

14. On lira avec profit le numéro spécial de Contrastes (Laurian 1987) et Bourquin (1993).

15. En réponse à une question de $M$. Perrin sur le fait que nous n'utilisons pas le terme acronyme, nous préférons en employant siglaison, la matrice lexicale décrite par J. Tournier, mettre l'accent sur le processus dynamique qui se traduit par des additions aux dictionnaires.

16. Outre les actes des colloques de Toulouse 1984, Paris Dauphine 1986 et Paris 5 IUT 1988, on se reportera à la présentation de la démarche de l'équipe Cobuild (Sinclair 1987).

17. Cette question mériterait un développement spécifique. Il est compréhensible que les médecins considèrent que l'emploi d'un terme médical par un non-médecin lui fait perdre de sa précision.

18. Elizabeth Jolivet pose la question des métaphores qui sont devenues des clichés. Il parait souhaitable que l'enseignant signale celles qui se sont figées, mais il ne semble pas toujours possible d'aboutir à un consensus. On peut espérer que la terminologie saura rapidement se doter d'une procédure appropriée qui ne se borne pas à l'argument d'autorité. Du strict point de vue de la création lexicale, que la métaphore se fige ou non, l'ajout au dictionnaire qu'elle représente doit être pris en compte.

19. Cf. note 14 .

\section{RÉSUMÉS}

Diverses contraintes pèsent sur l'organisation des cursus de langue anglaise des scientifiques. La montée en puissance d'une recherche spécifique en lexique et syntaxe paraît un des moyens d'assurer l'autonomie d'une didactique de terrain appropriée.

Curricula in English for Scientific Purposes have been handicapped by traditional decision making procedures. More research on the lexicon and syntax of scientific corpora should prove helpful in building up this recent field in English Language Teaching.

\section{INDEX}

Keywords : discourse analysis, lexicology, linguistics, language learning, scientific English Mots-clés : analyse du discours, anglais scientifique, didactique des langues, lexicologie, linguistique 


\section{AUTEUR}

\section{JEAN-LOUIS VIDALENC}

Faculté des Lettres, Université de Pau. jean-louis.vidalenc@orange.fr 